\title{
Modeling and Evaluation of a Policy Provisioning Architecture for Mobile Ad-Hoc Networks
}

\author{
Kaustubh S. Phanse, ${ }^{1,4}$ Aniket Bhat, ${ }^{2}$ and Luiz A. DaSilva ${ }^{3}$
}

\begin{abstract}
The ability to dynamically configure mobile ad-hoc network (MANET) devices is critical for supporting complex services such as quality of service (QoS), security and access control in these networks. In our work, we address the problem of policy distribution and provisioning in MANETs. Previously, we have proposed a policy provisioning architecture that enables the operator, e.g., a military command and control center, to maintain a logically centralized control of the network as a whole, while allowing a physically decentralized and self-managing implementation essential for a MANET environment. In this paper, we present an analytical model of the availability of the policy distribution service in a MANET and analyze the performance using stochastic Petri nets (SPNs). We develop the model at two levels of complexity: as a simple Markovian model with Poisson assumptions and as a more accurate nonMarkovian model with general distributions obtained using statistical parameterization. We compare and cross-validate the analytical results with simulation and experimental results. Finally, we illustrate the effectiveness of the architecture for managing QoS for soft real-time applications, using an emulated mobile ad-hoc network testbed.
\end{abstract}

KEY WORDS: Mobile ad-hoc network; policy-based network management; service availability; stochastic petri nets; simulation; testbed.

\section{INTRODUCTION}

Mobile ad-hoc networks (MANETs) have the potential to provide rapid, costeffective communication in situations where no central communication infrastructure (e.g., base station) exists or where deploying infrastructure is impractical.

\footnotetext{
${ }^{1}$ Luleå University of Technology, Department of Computer Science and Electrical Engineering, SE-971 87 Luleå, Sweden.

${ }^{2}$ Computer Networks and Software, Inc. 7405 Alban Station Ct, Suite B-215 Springfield, VA 22150, USA.

${ }^{3}$ Virginia Polytechnic Institute and State University, Bradley Department of Electrical and Computer Engineering 4300 Wilson Blvd. Ste. 750 Arlington, VA 22203, USA.

${ }^{4}$ To whom correspondence should be addressed at. E-mail: kphanse@ltu.se.
} 
The primary applications of MANETs, such as in battlefield scenarios and in disaster recovery operations, are often temporary and mission-centric, where nodes come together and cooperate to achieve certain objectives. Support and management of complex networking capabilities such as QoS, network security, and access control in these environments is mission critical. Many instances of reallife MANETs such as military networks also involve coalition-wide deployments where the MANET is a "network of networks" belonging to and managed by different organizations. This requires solutions that facilitate distributed, automated and self-organizing network management, while allowing a logically centralized federated control, e.g., from a military command and control center, of the network as a whole. We focus on the problem of policy distribution and network provisioning in such heterogeneous MANET environments. This task is significantly more challenging given that nodes may move across different subnetworks, requiring federated control policies to adapt, new nodes may join the network, at times replacing some of the existing nodes, and the roles played by the nodes may change over time to reflect changes in the networking conditions or the mission goals.

Policy distribution and network provisioning has been widely studied in the context of wired internetworks. Policy-based network management (PBNM) [1-4] has evolved from a centralized client-server model allowing an operator to control the network as a whole in an automated and simplified fashion. For implementing policies in an ad-hoc network environment in an efficient and scalable manner, the PBNM concept must first be adapted to a distributed paradigm suitable to MANETs. In our previous work [5, 6], we proposed a policy provisioning architecture for MANETs comprising the following solutions:

- A distributed service discovery mechanism to allow mobile nodes to discover the policy servers in the network with minimal additional overhead;

- $k$-hop clustering for localized and efficient management, where only those clients within $k$ hops from a policy server can obtain its service;

- Dynamic service redundancy (DynaSeR) techniques for invoking policy server instances on demand and improving policy service coverage; and

- Support of seamless implementation of federated control policies in a multi-organizational MANET via policy negotiation.

The implementations of DynaSeR and policy negotiation include extensions to the standard Common Open Policy Service for Provisioning (COPS-PR) protocol [7].

In this paper, we present a methodology for mathematical modeling of our policy distribution architecture using stochastic Petri nets, with particular focus on evaluating the architecture's service availability and the main factors that influence it. We cross-validate our findings based on the analytical model through comparison with simulation and experimental results. 
The remainder of this paper is organized as follows. In Section 2, we review relevant previous work and highlight the contribution of this paper. In Section 3, we present our analytical model and discuss parameterization of the model. In Section 4, we describe our experimental network testbed and compare results obtained from the experimental, simulation and analytical evaluation. We also demonstrate the effectiveness of the proposed architecture for QoS management in support of soft real-time applications in a MANET. Finally, Section 5 summarizes our conclusions.

\section{RELATED WORK}

Network monitoring and data collection have been the focus of most published work on ad-hoc network management. The Ad-hoc Network Management Protocol (ANMP), proposed by Chen et al. [8], uses an enhanced SNMP management information base (MIB-II) to perform data collection, fault management, and security management. However, ANMP still lacks the efficiency, reliability and robustness expected in a protocol for network provisioning [9, 10]. In [11], a Guerilla Management Architecture based on the concept of mobile agents is proposed as an alternative to the conventional client-server architecture. The focus is on monitoring network parameters such as battery power usage, processing load, and node isolation probability. While the approach in [11] seems promising, no quantitative performance analysis is presented. It is unclear how effectively the concept of mobile agents can be used for policy-based provisioning. Interoperability of such an approach with existing standard management protocols also needs to be better understood. In [12], Cheng and Chen motivate propose methods for mobility management - location tracking and handling of topology changes - in a mobile ATM network.

In recent times, there has been a growing interest in extending policy-based networking to mobile and nomadic computing. Munaretto et al. [13] discuss the adoption of policy-based management for mobile users within an enterprise network, while Harroud et al. [14] propose and demonstrate an agent-based architecture to provide policy-driven personalized multimedia services to nomadic users. Both works only consider infrastructure-based (e.g., wireless LANs, 3G) mobile networks. To our knowledge, PBNM has not been extensively studied in the context of ad-hoc networks. An agent-based architecture for policy-based adhoc network management is introduced in [15], but performance evaluation of the architecture is not reported.

In our previous work, we proposed a policy-based management framework [16] and listed the requirements for using PBNM in a MANET. A hybrid architecture - one that combines the outsourcing and provisioning techniques - was identified as a suitable approach for policy distribution in MANETs. In [5, 6], we proposed solutions for enabling efficient, reliable and scalable distribution of 
control policies in MANETs. In [6], we described our proof of concept experiments using a wireless ad-hoc network testbed; this preliminary study included a simple COPS-PR client-server architecture without our solution suite. [5] presented our complete policy provisioning architecture and a simulation-based evaluation of the architecture in different mobile ad-hoc networking conditions.

In this paper, we focus on analytical modeling and evaluation of the architecture in a MANET environment using stochastic Petri nets [17, 18]. Petri nets have been widely used to model various aspects of computer networks, e.g., [19-22]. We use stochastic Petri nets to develop analytical model of the service availability experienced by policy clients in our architecture. The model is developed at two levels of complexity: a simple Markovian model with Poisson assumptions and a detailed non-Markovian model, where we represent the system by the general distributions observed via statistical parameterization using simulation. We also conduct an experimental evaluation and compare the results obtained from analytical, simulation and experimental methods. Finally, we demonstrate the utility the proposed architecture for managing quality of service in multi-domain MANETs. The main contributions of this paper are:

- Analytical models that allow a more accurate characterization of the service availability of our proposed policy distribution architecture in a MANET environment and understanding the various components that influence it;

- Testbed experiments using our prototype implementation; and

- Cross-validation of the simulation, experimental and analytical results.

\section{MODELING SERVICE AVAILABILITY}

\section{Policy Provisioning Architecture}

Here, we briefly describe the key components of our policy provisioning architecture for mobile ad-hoc networks (MANETs). For a more detailed description of the architecture, we refer the reader to [9].

The architecture consists of policy servers that distribute policies to the client nodes in a mobile ad-hoc network. A $k$-hop clustering scheme limits the maximum number of hops between a policy server and client to $k$. The clustering scheme is motivated by our initial real-life testing of the COPS-PR protocol in a multihop wireless ad-hoc network [6]; it allows efficient and reliable policy distribution through localized service. A service discovery mechanism allows client nodes to discover policy servers in the network by listening to periodic service advertisement messages broadcast by server nodes or by broadcasting service request messages. We limit the service discovery messages to $k$-hop-limited broadcasts and propose a heuristic to considerably reduce the number of redundant messages without much computational overhead. Our Dynamic Service Redundancy 


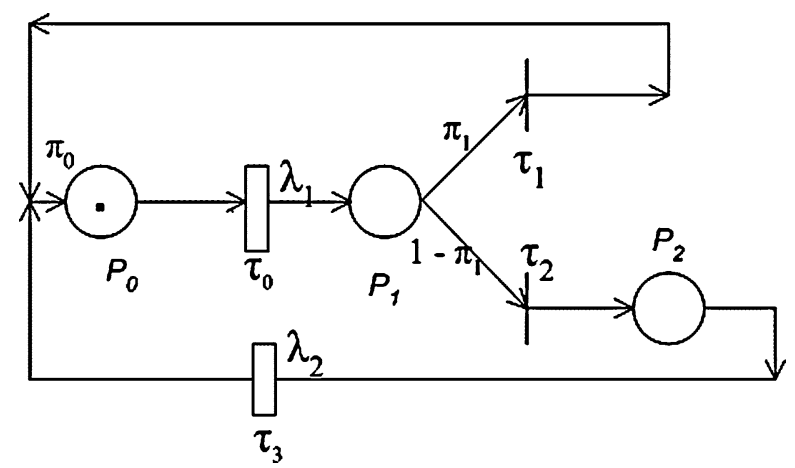

Fig. 1. GSPN model for service availability experienced by policy clients.

(DynaSeR) techniques improve the service coverage of the policy servers; it includes a novel delegation mechanism to invoke new service instances at suitable client nodes for dynamically adapting the service availability to topological changes. We propose extensions to the COPS-PR protocol to enable policy negotiation between policy servers administering different domains or subnetworks within a single coalition MANET.

In the following sub-sections, we develop analytical models for the service availability as experienced by policy clients in our policy distribution architecture. For simplifying the analysis, we currently do not account for DynaSeR and policy negotiation in our models.

\section{Markovian Generalized Stochastic Petri Net (GSPN) Model}

When a policy client is covered within one of the clusters in the network it is said to be in service, while when it is not covered within any of the clusters it is said to be out of service. Figure 1 shows the generalized stochastic Petri net (GSPN) model for service availability as perceived by a policy client in our system. A GSPN is a Petri net that makes Markovian assumptions for timed transitions, i.e., transitions follow an exponential distribution, and uses switching probabilities for immediate transitions [17].

The GSPN model consists of a set of places $P=\left\{P_{0}, P_{1}, P_{2}\right\}$ and a set of transitions $T=\left\{\tau_{0}, \tau_{1}, \tau_{2}, \tau_{3}\right\}$. A single token, initially in place $P_{0}$, represents the client in the system. The presence of a token in place $P_{0}$ represents that the client is within one of the $k$-hop clusters in the network, and the probability $\pi_{0}$ associated with that event represents the service availability experienced by the client. The initial marking of the GSPN model is assumed to be $M_{0}=(1,0,0)$. This initial placement of tokens can be changed without changing the results of the analysis of the model [17]. Note that there is just a single token, corresponding to a single 


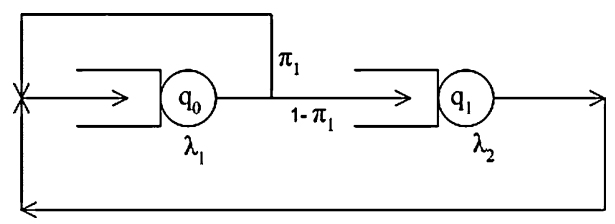

Fig. 2. Queuing model for service availability experienced by policy clients.

client under observation, but the analysis is generally applicable to any client in the system. The transition $\tau_{0}$ represents the client's movement out of a cluster. A rate of $\lambda_{1}$ is associated with the firing of that transition. A token in place $P_{1}$ represents that the client has moved out of the cluster; this is a vanishing marking [17]. It enables two immediate transitions $\tau_{1}$ and $\tau_{2}$, either of which can fire in accordance to the switching probabilities or weights associated with them. $\pi_{1}$ represents the probability with which the client moves immediately into a new cluster, once it moves out of its current cluster. This is the switching probability associated with the immediate transition $\tau_{1}$ and, hence, a switching probability of $1-\pi_{1}$ is associated with the immediate transition $\tau_{2}$. A token in place $P_{2}$ represents the scenario where a client, upon moving out of a cluster, has not immediately moved into a new cluster. The transition $\tau_{3}$ represents the time it takes for this "out of service" client to enter a new cluster. A rate of $\lambda_{2}$ is associated with the firing of transition $\tau_{3}$. We used the Stochastic Petri Net Package (SPNP) [23] to solve the GSPN model shown in Fig. 1. The results are discussed in Section 4.

We also developed the equivalent closed network of queues model, shown in Fig. 2. The network of queues model consists of two queuing systems $q_{0}$ and $q_{1}$. The queuing system $q_{0}$ represents the state that the client is currently within $k$ hops of some server in the system and, hence, in service. The service time of the queuing system $q_{0}$ thus represents the amount of time for which the client remains inside a $k$-hop cluster. When the client leaves its current cluster, it either moves back into a new cluster with a probability $\pi_{1}$ or enters the queuing system $q_{1}$. The queuing system $q_{1}$ represents the time for which client remains out of service. The service rates of the two queuing systems $q_{0}$ and $q_{1}$ are therefore $\lambda_{1}$ and $\lambda_{2}$ transitions/second, respectively. We solve the system using the Gordon-Newell algorithm for closed network of queues [24]. The service availability, given by the probability that there is one client in system $q_{0}$ and zero clients in system $q_{1}$, can be expressed as follows (we omit the simple derivation):

$$
p(1,0)=\frac{\lambda_{2}}{\lambda_{2}+\left(1-\pi_{1}\right) \lambda_{1}}
$$




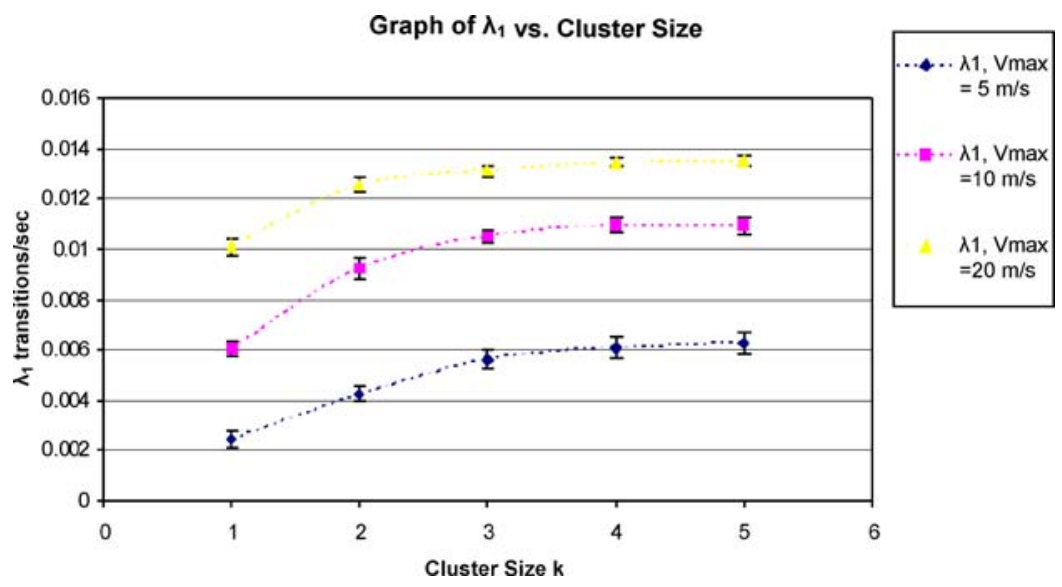

Fig. 3. Effect of mobility and cluster size on $\lambda_{1}$.

\section{Empirical Parameterization}

For a more accurate analysis of the client behavior and to validate whether our previous Markovian assumptions hold true, we conducted an empirical study of the various parameters of interest: $\lambda_{1}$ or the rate at which a client moves out of a cluster, $\lambda_{2}\left(1-\pi_{1}\right)$ or the rate at which a client moves back into a service cluster after spending some time without service, and $\pi_{1}$ or the probability that a client has immediately moved into a service cluster after leaving its existing cluster. We used the QualNet ${ }^{\mathrm{TM}}$ network simulator [25] for this purpose. The simulations used our implementation of the COPS-PR protocol and the built-in QualNet models for the OLSR ad-hoc routing protocol and 802.11b MAC layer. The simulated environment consisted of a $1000 \times 1000$ square meter flat area. We employ the commonly used Random Waypoint mobility model [26]. Three different mobility scenarios $\left(V_{\max }=5 \mathrm{~m} / \mathrm{s}, 10 \mathrm{~m} / \mathrm{s}\right.$, and $20 \mathrm{~m} / \mathrm{s}$ ) were considered. The minimum speed $\left(V_{\min }\right)$ parameter was set to around $90 \%$ of the maximum speed $\left(V_{\max }\right)$ for all simulations. This avoided the speed-decay problem reported in [27] and also helped reach steady state in an acceptable time (less than $500 \mathrm{~s}$ ). Pause time was set to $10 \mathrm{~s}$. Each simulation scenario is run for $1500 \mathrm{~s}$; we disregard data in the 500-second "warm-up" period. The COPS Keep-Alive (KA) message interval was set to $50 \mathrm{~s}$. Several runs of each simulation scenario were conducted to obtain the average values with $95 \%$ confidence intervals, each simulation run using a random initial placement of nodes.

Figure 3 shows the effect of varying mobility and cluster size $k$ on the average value of $\lambda_{1}$. As its speed increases, a node spends less time in a cluster, resulting in an increase in $\lambda_{1}$ with increased mobility. Also, $\lambda_{1}$ increases with cluster size. We 
Phanse et al.

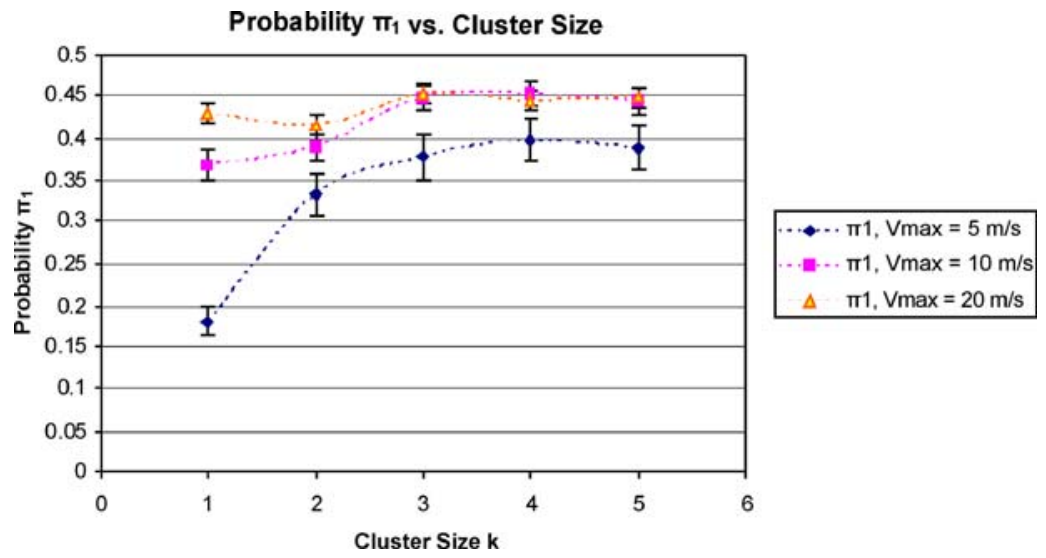

Fig. 4. Effect of mobility and cluster size on $\pi_{1}$.

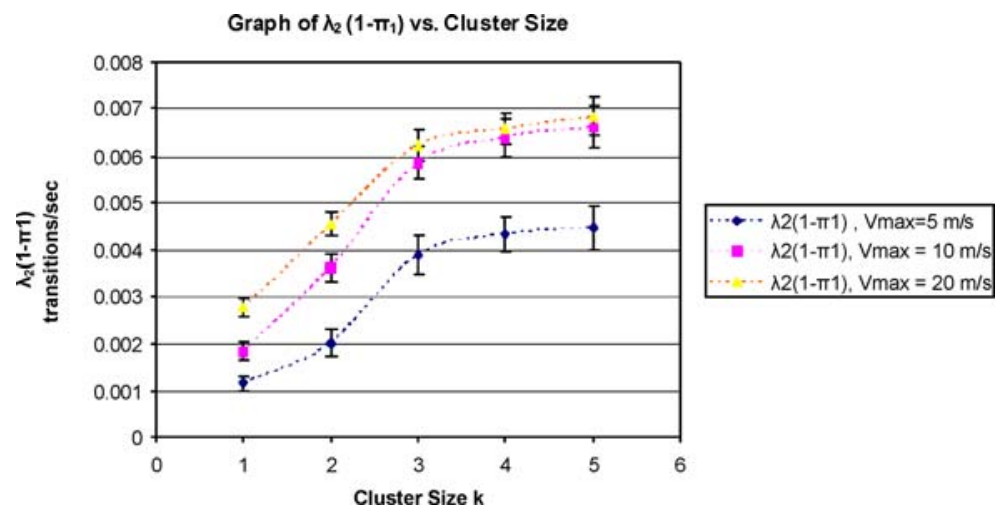

Fig. 5. Effect of mobility and cluster size on $\lambda_{2}\left(1-\pi_{1}\right)$.

attribute this to the fact that in presence of larger clusters, clients find themselves moving within $k$ hops of new cluster-heads more often.

Figure 4 shows the effect of varying speed of mobile nodes and cluster size $k$ on the estimated value of the probability $\pi_{1}$ that the clients move into a new cluster immediately after moving out of their current clusters. As the cluster size increases, the likelihood of a client getting covered within $k$ hops of a cluster-head increases. Also, with increase in node speeds, clients move into new clusters more quickly, and hence $\pi_{1}$ increases with mobility. The effect of mobility is most evident for the smallest cluster size of $k=1$.

In Fig. 5, we plot the variation of the product $\lambda_{2}\left(1-\pi_{1}\right)$ versus different mobility values and cluster sizes. The results match our intuition that the product 
Histogram of Times Spent By Clients Inside a Cluster (Reciprocal of $M_{1}$ )

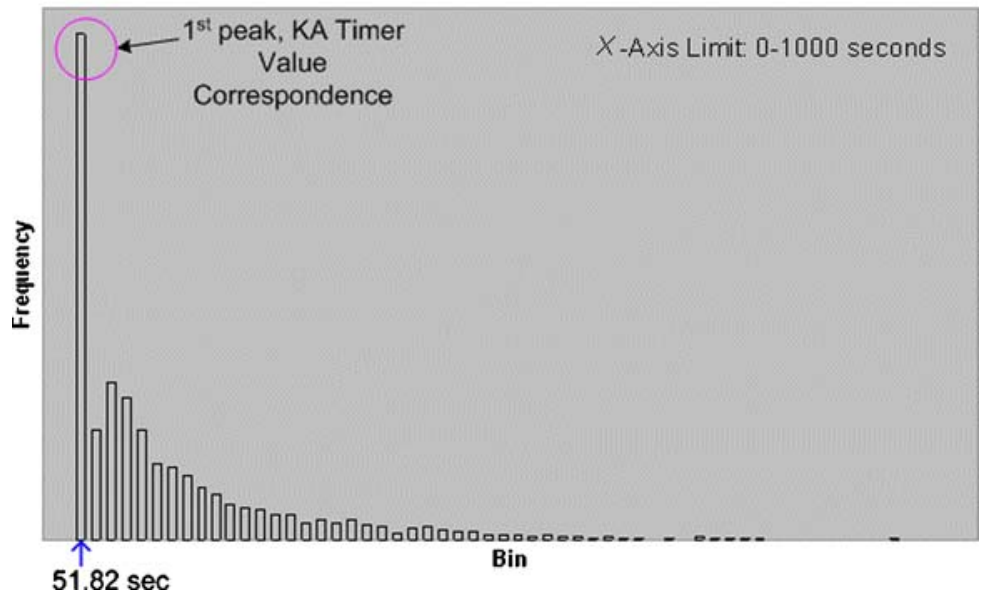

Fig. 6. Histogram of times spent by clients inside a cluster $\left(V_{\max }=10 \mathrm{~m} / \mathrm{s}, k=3\right)$.

value increases with increase in mobility as well as with increase in cluster size. This is attributed to the fact that clients stay out of service for a shorter duration of time and are eventually covered in another cluster.

We also gained insight into the nature of the processes associated with these parameters by observing the distribution of the instantaneous values of times spent by clients in and out of a cluster. These are essentially the reciprocal of instantaneous values of rates $\lambda_{1}$ and $\lambda_{2}\left(1-\pi_{1}\right)$, respectively.

Figure 6 shows the histogram of time spent by clients inside a cluster for a sample scenario wherein $V_{\max }=10 \mathrm{~m} / \mathrm{s}$ and cluster size $k=3$. The histogram follows a bi-modal distribution: the first peak is due to the expiration of the COPS Keep-Alive (KA) timer associated with each COPS connection. Whenever a client's KA timer expires, the client perceives that the server is unreachable and hence closes the COPS connection. If the client does not lose connectivity due to expiration of the KA timer, it follows a general distribution of time to exit its cluster. TTThe bimodal behavior with the first peak characterized by the KA timer was consistently observed across all the scenarios we studied.

Figure 7 shows the histogram of the time spent by clients out of any cluster (the reciprocal of $\lambda_{2}\left(1-\pi_{1}\right)$ ) for $V_{\max }=10 \mathrm{~m} / \mathrm{s}$ and $k=3$; it approximately follows a uniform distribution. This behavior was observed for all other scenarios considered. Since the probability $\pi_{1}$ is a scalar value, the histogram of $\lambda_{2}$ will be a scaled version of the one shown in Fig. 7. 


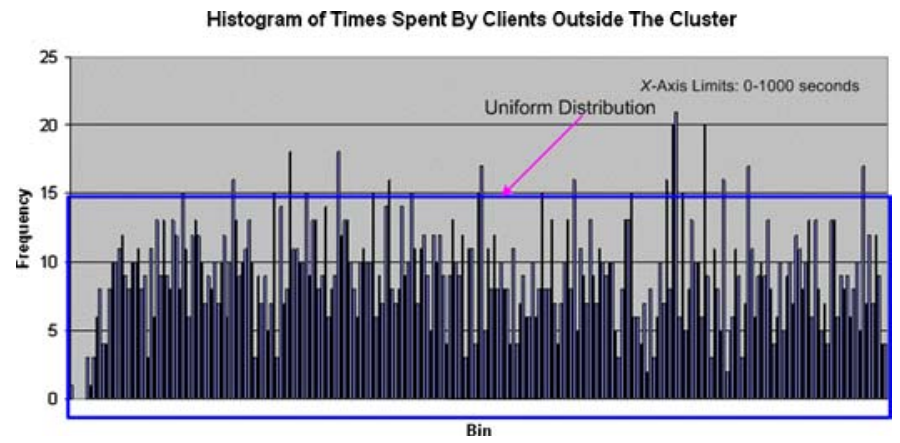

Fig. 7. Histogram of times spent by clients outside all clusters $\left(V_{\max }=10 \mathrm{~m} / \mathrm{s}, k=3\right)$.

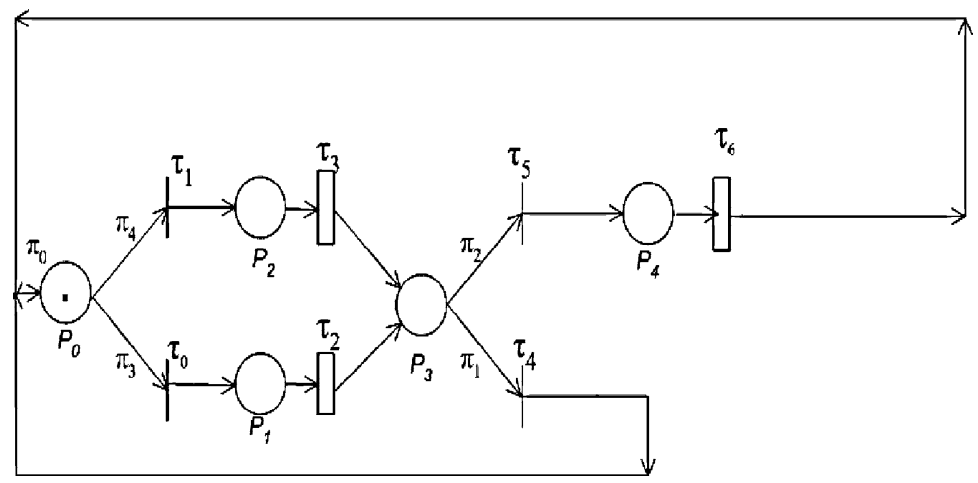

Fig. 8. GDT_SPN model for service availability experienced by policy clients.

\section{Non-Markovian Generally Distributed Transition Stochastic Petri Net (GDT_SPN)}

From the statistical analysis in Section 3.3, it is evident that our previous assumption about the reciprocals of $\lambda_{1}$ and $\lambda_{2}$ following an exponential distribution does not hold true. We enhance our GSPN model (Fig. 1) to account for the bimodal and uniform distributions observed in the simulation. The new model, namely the generally distributed transition stochastic Petri net (GDT_SPN) model, is shown in Fig. 8.

The model consists of a set of places $P=\left\{P_{0}, P_{1}, \ldots, P_{4}\right\}$ and a set of transitions $\mathrm{T}=\left\{\tau_{0}, \tau_{1}, \ldots, \tau_{6}\right\}$. As in the Markov model, we have a single token representing a particular client in the system. Unlike the GSPN model, the presence of a token in place $P_{0}$ represents a vanishing condition and the token immediately gets transferred to either place $P_{1}$ or place $P_{2}$. The presence of the token in any of the three places signifies that the client is currently being covered inside some 
cluster and, hence, is in service. The bi-modal distribution of the time that a client remains in a cluster can be characterized by the combination of a deterministic time transition and a generally distributed time transition. Hence, we split the transition $\tau_{0}$ of Fig. 1 into two transitions $\tau_{2}$ and $\tau_{3}$. Note that transition $\tau_{2}$ models the movement of clients out of a cluster caused by the expiration of the KA timer. Transition $\tau_{3}$ is used to model the other case of clients moving out after a generally distributed time shown in Fig. 6. The immediate transitions $\tau_{0}$ and $\tau_{1}$ and the dummy places $P_{1}$ and $P_{2}$ are used to model the switching probabilities for transitions $\tau_{2}$ and $\tau_{3}$. Thus, $\pi_{3}$ is the probability with which a client leaves its cluster after a fixed time (modeled by transition $\left.\tau_{2}\right)$ and $\pi_{4}=\left(1-\pi_{3}\right)$ is the probability with which the client moves out of the cluster after a generally distributed time. Place $P_{3}$ serves as an output place for the two transitions $\tau_{2}$ and $\tau_{3} . \pi_{1}$ represents the probability that a client moves into a new cluster immediately after departing its original cluster, and $\pi_{2}$ is simply $\left(1-\pi_{1}\right)$. If the client does not move into a new cluster immediately, then it enters the state where it is looking for service. This state is represented by a token in place $P_{4}$. We employ transition $\tau_{6}$ to represent the uniform distribution that approximates the random variable associated with the time spent by clients outside any cluster.

As the processes involved in the model depart from the simplistic Poisson assumptions, closed-form mathematical representation for steady state probabilities becomes infeasible. In particular, we identified the transition $\tau_{3}$ in Fig. 8 to be a generally distributed transition, meaning it cannot be approximated to any of the known standard distributions. Hence, unlike the Markovian analysis where we are able to apply queuing theory results to validate the GSPN model, in the non-Markovian analysis of our model, we resorted to a multi-stage approximation method for analyzing the complex process model of service availability. Here, we briefly describe the steps taken. For a more detailed discussion on the methodology, we refer the reader to [28]. We used the WebSPN tool [29] for model specification. To specify generally distributed transitions in WebSPN, it is necessary to approximate the general distributions associated with these transitions to discrete phase type distributions. We used a tool called PhFit [30] for this purpose. The PhFit analysis was performed for transition $\tau_{3}$ using a discretization step $\delta=1$ and the number of phases $n=8$. This approximation was done for all mobility scenarios with cluster size varying from $k=1$ to $k=5$. The output of the PhFit tool is the acyclic discrete phase type (ADPH) distribution approximation of the general distribution in the Canonical Form 1 (CF1). Bobbio et al. define a canonical form representation as a unique minimal representation of the ADPH distribution [31]. For the specification of DPH transitions in WebSPN, it is essential to convert the results obtained to the Canonical Form 2 (CF2) or Canonical Form 3 (CF3) [31]. We converted the $\mathrm{CF} 1$ output of the PhFit tool into the CF2 form [30] as a matrix suitable as an input to the WebSPN tool. 
Phanse et al.

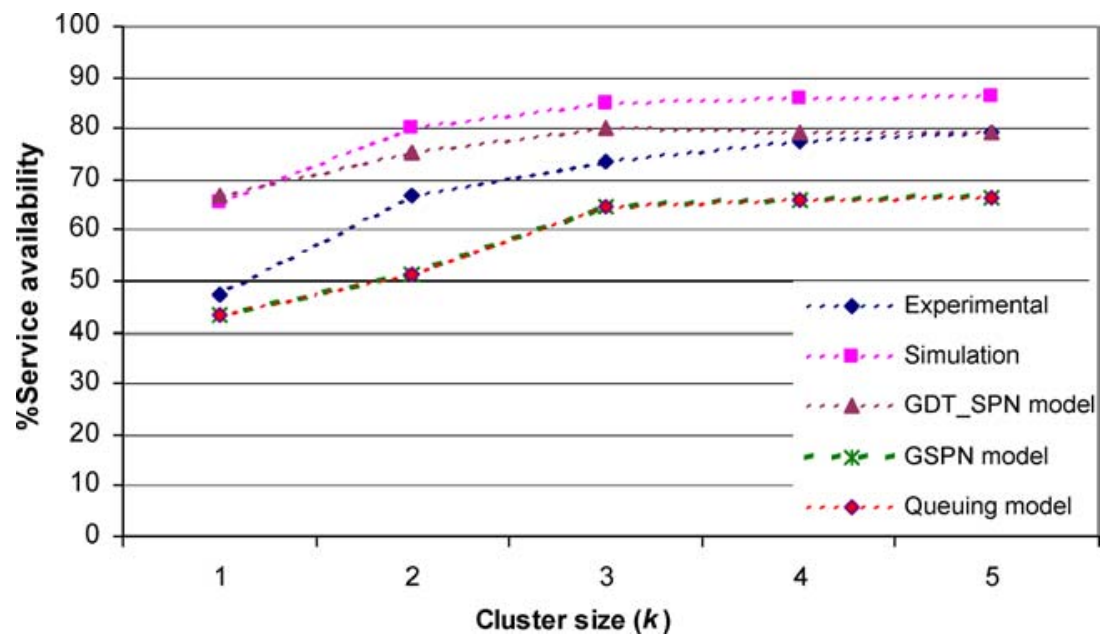

Fig. 9. Comparison of the service availability results obtained using different methods.

\section{ANALYTICAL AND EXPERIMENTAL EVALUATION}

\section{Cross-Validation}

In addition to the analytical models developed in this paper, we conducted experimental evaluation with the purpose of testing our prototype implementation of the policy provisioning architecture, and verifying the trends exhibited in the analytical results and simulation results. To conduct repeatable experiments, we used a wired network testbed comprised of ten Linux-based machines. The mobility of nodes in the network and the resulting topological changes was emulated using the Dynamic Switch [32] software. BonnMotion [33], a Java-based software, was used to create mobility trace files following the Random Waypoint model with settings identical to those in the simulation study. The OLSR protocol was used for ad-hoc routing. Several runs for each scenario (cluster size and node speed) were considered to calculate the average service availability.

Figure 9 shows the average service availability $\left(V_{\max }=10 \mathrm{~m} / \mathrm{s}\right)$ as a function of cluster size, obtained from five different methods: GSPN model (Fig. 1) solved using SPNP, closed network of queues model (Fig. 2), GDT_SPN model (Fig. 8), simulation using QualNet, and experiments conducted using the emulated mobile ad-hoc network testbed. The results from the GSPN model exactly match the results from our queuing model. While the general trend of these curves seems to match the trend of the simulation and experimental curve, there is considerable difference in the values for service availability. On relaxing the Markovian assumption and with the more accurate distributions in place (a bimodal distribution 
approximation for $\lambda_{1}$ and a uniform distribution approximation for $\lambda_{2}$ ), the results from the GDT_SPN model match the simulation and experimental results well.

\section{Illustration: QoS Management for Soft Real-Time Applications}

Thus far, we modeled and characterized the performance of our architecture in a mobile ad-hoc network environment. In this section, we demonstrate the architecture at work, with specific application to dynamic bandwidth management in a multi-domain mobile ad hoc internetwork such as a military coalition MANET. In particular, this exemplifies the effectiveness of our proposed COPS-PR based policy negotiation mechanism to provide seamless QoS in such networks. To our knowledge, only other work that addresses dynamic service negotiation in mobile environments is the Dynamic Service Negotiation Protocol (DSNP) [34]. Four different administrative domains were setup using the emulated MANET testbed. A real-time application software [35] was used to generate time-sensitive traffic. The Linux traffic control tool was used to implement traffic classification and scheduling. Random node mobility was emulated using the Dynamic Switch. To fairly characterize the effectiveness, we ensured multi-hop connectivity between the application endpoints during the experiment. Transmission of three traffic flows from a source node was considered: two soft real-time applications with distinct utility functions (each application transmitting at the rate of $48 \mathrm{~kb} / \mathrm{s}$ ) and one flow representing background traffic (data rate of about $32 \mathrm{~kb} / \mathrm{s}$ ). The source node moved randomly across the various mobile domains. Three metrics were considered: percentage accrued utility, percentage missed deadlines and average throughput. The performance of the two real-time applications was captured using the monitoring tool and choirGUI (part of the real-time software package [35]).

As seen in Fig. 10, the GUI consists of four windows. The window on the bottom-right shows the utility functions for the two real-time applications. The $\mathrm{X}$-axis represents the end-to-end delay and the $\mathrm{Y}$-axis represents the utility. The end-to-end delay for each received packet is recorded and indicated by vertical bars appearing in this window (shifting along the $\mathrm{X}$-axis as the delay changes). Whenever a packet is received after the deadline - the threshold delay beyond which the utility for that application is zero - the corresponding bars appear red, indicating a missed deadline. The top-right window shows the total accrued utility as percentage of the maximum utility. The window on the top-left displays the percentage of missed deadlines, while the window on the bottom-left shows the average throughput for the real-time applications as well as the background traffic. Figure 10 shows the case without policy negotiation. Initially, the source node is resident in its home domain and obtains the desired QoS for the real-time applications. As the source node moves into foreign domains, the real-time traffic is classified along with the background into the default best-effort class (due to lack of appropriate QoS policies), leading to considerable decrease in throughput. 


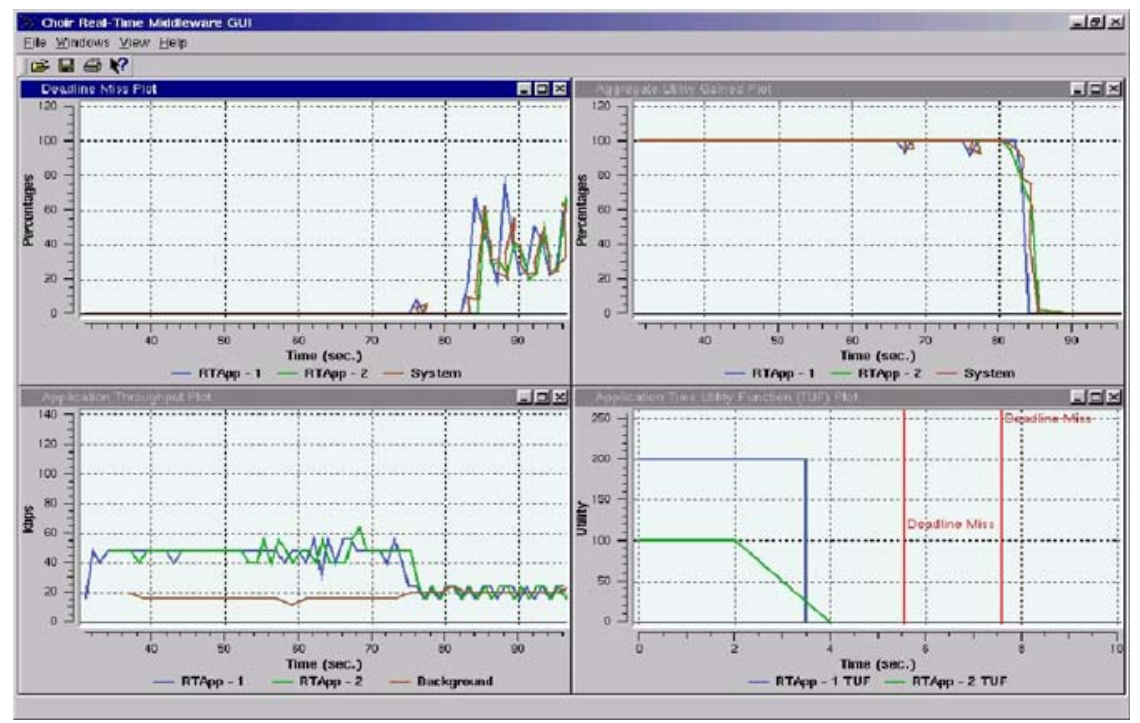

Fig. 10. Without policy negotiation, the real-time mission critical applications are treated as best-effort along with background traffic as the source node moves into foreign domains.

Soon the end-to-end packet delay increases beyond the threshold, and packets start missing deadlines as shown by the vertical bars in the bottom-right window. The percentage accrued utility (seen in top-right window) eventually drops to zero.

In presence of the policy negotiation mechanism, the various domains are able to negotiate policies on-the-fly and the node receives the desired QoS for its realtime applications as it moves into domains administered by other organizational policies. The resulting plot captured using the choirGUI is shown in Fig. 11. Except for the few temporary glitches in the performance (e.g., around the 100, 170,210 and $250 \mathrm{~s}$ marks) when the node moves from one domain to another, the real-time applications experience almost seamless QoS.

\section{CONCLUDING REMARKS}

In this paper, we presented a methodology using stochastic Petri nets for analytical modeling of a policy distribution architecture for MANETs. In particular, we focused on evaluating the service availability of the architecture. The analytical model was developed and evaluated once with Markovian assumptions and then by relaxing those assumptions based on the statistical analysis of the key parameters in the model. Assuming exponentially distributed parameters led to a simple model that could be evaluated using queuing theory. However, the results in that 


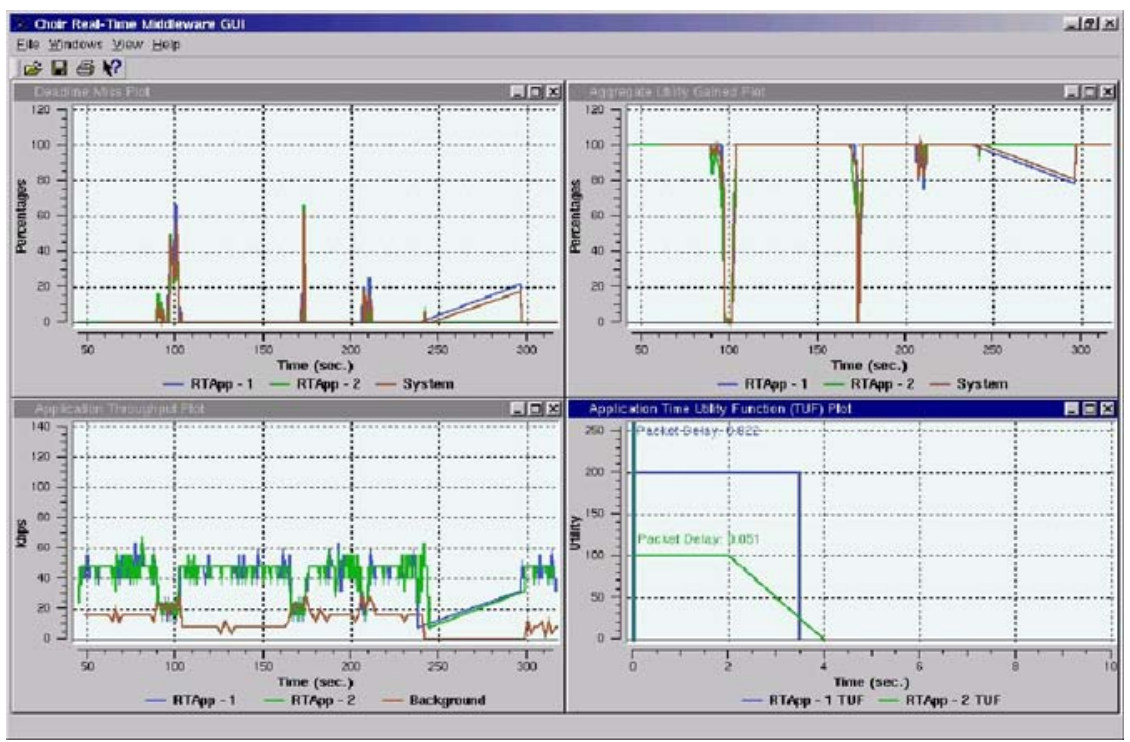

Fig. 11. Almost seamless QoS is achieved for real-time mission critical applications in presence of policy negotiation as the source node moves across different network domains.

case did not match the experimental and simulation results. Taking into account more accurate distribution of the significant parameters, observed via empirical methods, resulted in a complex model requiring more sophisticated applied techniques for solving. The results obtained from this improved model specification matched well with the simulation and experimental results. We demonstrated the effectiveness of the proposed architecture for managing quality of service for soft real-time applications through experiments conducted using an emulated mobile ad-hoc network testbed.

\section{ACKNOWLEDGEMENTS}

The work presented in this paper was partly funded by the Office of Naval Research as a part of the Navy Collaborative Integrated Information Technology Initiative (NAVCIITI). We would like to thank the anonymous reviewers for their useful comments for improving this manuscript.

\section{REFERENCES}

1. D. Verma, Policy-Based Networking: Architectures and Algorithms, New Riders Publishing, 2000. 
2. D. Kosiur, Understanding Policy-Based Networking, John Wiley \& Sons, Inc., 2001.

3. Y. Kanada and B. O'Keefe, Rule-based Building-block Architectures for Policy-based Networking, Journal of Network and Systems Management, vol. 11, no. 3, September 2003.

4. L. Lymberopoulos, E. Lupu, and M. Sloman, An Adaptive Policy-based Framework for Network Services Management, Journal of Network and Systems Management, vol. 11, no. 3, September 2003.

5. K. Phanse and L. DaSilva, Protocol Support for Policy-Based Management of Mobile Ad Hoc Networks, Proceedings of IEEE/IFIP Network Operations and Management Symposium, pp. 3-16, April 2004.

6. K. Phanse, L. DaSilva and S. Midkiff, Design and Demonstration of Policy-Based Management in a Multi-Hop Ad Hoc Network Testbed, International Journal of Ad Hoc Networks, pp. 389-401, May 2005.

7. K. Chan et al., COPS usage for Policy Provisioning (COPS-PR), IETF RFC 3084, March 2001.

8. W. Chen, N. Jain, and S. Singh, ANMP: Ad Hoc Network Management Protocol, IEEE Journal on Selected Areas of Communications, vol. 17, no. 8, pp. 1506-1531, August 1999.

9. K. Phanse, Policy-Based Quality of Service Management in Wireless Ad Hoc Networks, Ph.D. dissertation, Electrical and Computer Engineering Department, Virginia Polytechnic Institute and State University, August 2003.

10. E. Liden and A. Torger, Implementation and Evaluation of the Common Open Policy Service (COPS) Protocol and its use for Policy Provisioning, Master's thesis, Department of Computer Science and Electrical Engineering, Luleå University of Technology, Sweden, January 2000.

11. C. Shen, C. Srisathapornphat, and C. Jaikaeo, An Adaptive Management Architecture for Ad Hoc Networks, IEEE Communications Magazine, vol. 41, no. 2, pp. 108-115, February 2003.

12. S. Cheng and C. Chen, Location and Configuration Management in Mobile-Switch ATM Networks, Journal of Network and Systems Management, vol. 9, no. 4, December 2001.

13. A. Munaretto, N. Agoulmine and M. Fonseca, Policy-based Management of Ad Hoc Enterprise Networks, Proceedings of the Workshop of the HP OpenView University Association, June 2002.

14. H. Harroud, M. Ahmed and A. Karmouch, Policy-Driven Personalized Multimedia Services for Mobile Users, IEEE Transactions on Mobile Computing, vol. 2, no. 1, January-March 2003.

15. R. Chadha, C. Chiang, M. Little and S. Samtani, Agent-Based Policy-Enabled Network Management Architecture for Mobile Ad Hoc Networks, Proceedings of IEEE Military Communications Conference, October 2003.

16. K. Phanse and L. DaSilva, Addressing the Requirements of QoS Management in Wireless Ad Hoc Networks, International Journal on Computer Communications, vol. 26, no. 12, pp. 1263-1273, July 2003.

17. W. Reisig, et al., Petri Nets 2000-Introductory Tutorial, Proceedings of the International Conference on Applications and Theory of Petri Nets, June 2003.

18. J. L. Peterson, Petri Net Theory and the Modeling of Systems, Prentice Hall Inc., 1981.

19. E. Gressier, A Stochastic Petri Net Model for Ethernet, Proceedings of the International Workshop on Timed Petri Nets, IEEE Computer Society Press, pp. 296-306, 1986.

20. K. S. Trivedi and H. Sun, Stochastic Petri Nets and Their Applications to Performance Analysis of Computer Networks, Proceedings of the International Conference on Operational Research for a Better Tomorrow, December 1998.

21. R. German and A. Heindl, Performance Evaluation of IEEE 802.11 Wireless LANs with Stochastic Petri Nets, Proceedings of the International Workshop on Petri Nets and Performance Models, pp. 44-53, September 1999.

22. C. Xiong, T. Murata, and J. Tsai, Modeling and Simulation of Routing Protocol for Mobile Ad hoc Networks Using Colored Petri Nets, Proceedings of Conference on Application and Theory of Petri Nets: Formal Methods in Software Engineering and Defense Systems, pp. 145-153, 2002.

23. Stochastic Petri Net Package. http://www.ee.duke.edu/ chirel/MANUAL/manual.pdf. 
24. W.J. Gordon and G.F. Newell, Closed Queuing Systems With Exponential Servers, Operations Research, vol. 15, pp. 254-265, 1967.

25. QualNet Network Simulator. http://www.scalable-networks.com.

26. J. Broch, D. Maltz, D. Johnson, Y. Hu, and J. Jetcheva, A performance comparison of multihop wireless ad hoc network routing protocols, Proceedings of ACM Mobile Computing and Networking, pp. 85-97, 1998.

27. J. Yoon, M. Liu, and B. Noble, Random Waypoint Considered Harmful, Proceedings of the $22^{\text {nd }}$ Annual Joint Conference of the IEEE Computer and Communications Societies, vol. 2, pp. 1312-1321, March 2003.

28. A. Bhat, Stochastic Petri Net Models of Service Availability in a PBNM System for Mobile Ad Hoc Networks, Master's thesis, Electrical and Computer Engineering Department, Virginia Polytechnic Institute and State University, 2004.

29. A. Puliafito, A. Bobbio, M. Scarpa, and M. Telek, WebSPN: A Web-accessible Petri Net tool, Proceedings of the conference on Web-based Modeling \& Simulation, January 1998.

30. A. Horváth, A. Bobbio, and M. Telek, PhFit: A General Phase-type Fitting Tool, Proceedings of the International Conference on Computer Performance Evaluation, Modeling Techniques and Tools, pp. 82-91, April 2002.

31. A. Bobbio, A. Horváth, M. Scarpa, and M. Telek, Acyclic Discrete Phase Type Distributions: Properties and a Parameter Estimation Algorithm, Performance Evaluation, vol. 54, no. 1, pp. 1-32, 2003.

32. T. Lin, S.F. Midkiff, and J.S. Park, A Dynamic Topology Switch for the Emulations of Wireless Mobile Ad Hoc Networks, Proceedings of the Annual IEEE Conference on Local Computer Networks, pp. 791-798, November 2002.

33. BonnMotion tool. Available at http://web.informatik.uni-bonn.de/IV/Mitarbeiter/dewaal/ BonnMotion/.

34. J-C Chen et al., Dynamic Service Negotiation Protocol (DSNP), IETF Internet Draft, work in progress, November 2004. http://www.ietf.org/internet-drafts/draft-itsumo-dsnp-02.txt.

35. K. Channakeshava, Utility Accrual Real-time Channel Establishment in Multi-hop Networks, M.S. thesis, Electrical and Computer Engineering, Virginia Tech, 2003.

Kaustubh S. Phanse is an Assistant Professor at the Department of Computer Science and Electrical Engineering, Luleå University of Technology, Sweden. He received his M.S. and Ph.D. in Electrical Engineering at Virginia Tech's Bradley Department of Electrical and Computer Engineering in 2000 and 2003, respectively and his B.E. in Electronics and Telecommunication from University of Mumbai, India, in 1998. His research interests and funded projects are in the areas of of delay/disruption tolerant networking, quality of service in mobile ad-hoc networks, self-positioning in wireless networks and performance of wireless LANs in harsh environments. He is a member of the IEEE and recipient of the Swedish Foundation for International Cooperation and Higher Education (STINT) scholarship for 2004 .

Aniket A. Bhat is a Systems Engineer with Computer Networks and Software, Inc in Springfield, Virginia. He received his Masters Degree in Computer Engineering from Virginia Tech in Summer 2004 and received his Bachelors in Electronics Engineering from University of Mumbai, India, in August 2001. He has been working in the area of computer networks for the past 4 years and has a thesis in analytical modeling of ad hoc networks. He is currently working on investigating security issues in the GMPLS control plane.

Luiz A. DaSilva is an Associate Professor at Virginia Tech's Bradley Department of Electrical and Computer Engineering. He received his Ph.D. in Electrical Engineering at the University of Kansas and previously worked for IBM for six years. His research interests focus on performance and resource management in wireless mobile networks and QoS issues. He is currently involved in funded research 
Phanse et al.

projects in the areas of QoS interoperability and policy-based network management, application of game theory to model mobile ad-hoc networks (MANETs), heterogeneous MANETs employing smart antennas, and cognitive networks. He has published over fifty refereed papers in journals and major conferences in the communications and computer areas. He is a senior member of IEEE and a member of ASEE. 